\title{
Detektion des Kartoffelspindelknollen Viroids mit Hilfe der Loop Mediated Isothermal Amplification
}

\author{
Katja Schulze, Jörn Glökler, Marianne Kadolsky, Marcus Frohme
}

Zusammenfassung

PSTV ist ein hoch infektiöses Viroid, das in Kartoffeln verkleinerte und spindelähnliche Knollen verursacht. Um Ernteverlusten vorzubeugen, ist eine Detektion in frühen Infektionsstadien von großer Bedeutung. Aufgrund ihrer hohen Sensitivität und Spezifität wird die PCR als Standardnachweisverfahren für PSTV verwendet. Nachteilig an dieser Methode sind der apparative Aufwand und die zeitaufwändige Durchführung.

Als viel versprechende Alternative konnte der Nachweis von PSTV mit Hilfe der Reverse Transcription Loop Mediated Isothermal Amplification (RT-LAMP) gezeigt werden. Hierbei handelt es sich um eine einfache und schnelle Methode, für die wenig aufwändige Laborausrüstung benötigt wird. Dabei ermöglichte eine an den Amplifikationsprozess gekoppelte Fluoreszenzreaktion die Detektion von Produkten direkt nach der Nachweisreaktion mit dem bloßen Auge (bzw. unter UV-Licht). Die Ergebnisse konnten mit Hilfe einer Real Time Detektion des auftretenden Fluoreszenzsignals bestätigt werden.

\section{Abstract}

PSTV is a highly infectious viroid which leads to small and spindle shaped tubers in potatoes. The detection at an early stage of infection is important to minimize loss of harvest. Normally PCR is used for the detection of PSTV, because of its high sensitivity and specificity. Disadvantages of this method are the requirement of sophisticated equipment and the time consuming process.

As a promising alternative, the detection of PSTV with the reverse transcription loop mediated isothermal amplification (RT-LAMP) was shown. LAMP is a very fast and simple detection method requiring only standard laboratory equipment. A fluorescence reaction, coupled to the amplification process, allowed the detection of amplification products directly after the reaction with the naked eye (or under UV light, respectively). Real time detection of the occurring fluorescence signal was possible and confirmed the obtained results.

\section{Einleitung}

Das Kartoffelspindelknollen Viroid (PSTV - Potato spindle tuber viroid) ist ein zirkuläres, einzelsträngiges RNA-Molekül von 359 Nukleotiden Länge mit charakteristischer Faltung, das die Spindelknollensucht in Kartoffeln hervorruft [1]. Hauptsymptom ist die Bildung von kleinen spindelförmigen Kartoffelknollen. Dies kann zu großen Ernteverlusten führen und stellt eine ökonomische Bedrohung dar. PSTV kann neben der Kartoffel auch andere Mitglieder der Familie der Nachtschattengewächse infizieren. Es ist hoch infektiös und kann sowohl mechanisch als auch durch Insekten übertragen werden $[2,3]$.

Für die Detektion des Viroids wurden verschiedene Methoden entwickelt; $u$. a. die Hybridisierung mit Digoxigenin gelabelten RNA Molekülen [4] und die Isolierung von Viroiden mit Hilfe der Return Polyacrylamid
Gelelektrophorese [5]. Aufgrund seiner hohen Sensitivität ist das heutige Standardverfahren ein RT-PCR Protokoll, welches von Weidemann und Buchta entwickelt wurde [6]. Für eine parallele Detektion von weiteren Pflanzenpathogenen wurde die Multiplex RT-PCR eingeführt [7, 8]. Mit Hilfe der Multiplex-ELISA-PCR [9] können Amplifikationsprodukte durch eine enzymatische Farbreaktion detektiert werden. Die Detektion von Amplifikationsprodukten während der PCR wird durch einen Real Time RT-PCR Ansatz möglich [10].

Nachteil aller bestehenden Methoden ist, dass entweder eine nicht ausreichende Sensitivität erreicht wird oder dass teure Spezialausrüstung wie Thermocycler, Gelelektrophorese System oder Real Time PCR Gerät benötigt werden. Eine viel versprechende Alternative hierzu stellt die loop mediated isothermal amplification (LAMP) dar [11]. Die Methode ist leicht durchzuführen, benötigt nur einfache Laborgeräte und ermöglicht die 
Amplifikation einer Zielsequenz unter isothermalen Bedingungen mit hoher Effizienz und Geschwindigkeit. Eine hohe Spezifität wird durch 4 verschiedene Primer gewährleistet, die an 6 spezifische Regionen der Zielsequenz binden (Abb. 1). Die Verwendung der Bst Polymerase, die eine Strangverdrängungsaktivität besitzt, ermöglicht eine Amplifikation bei gleich bleibender Temperatur $[11,12]$.

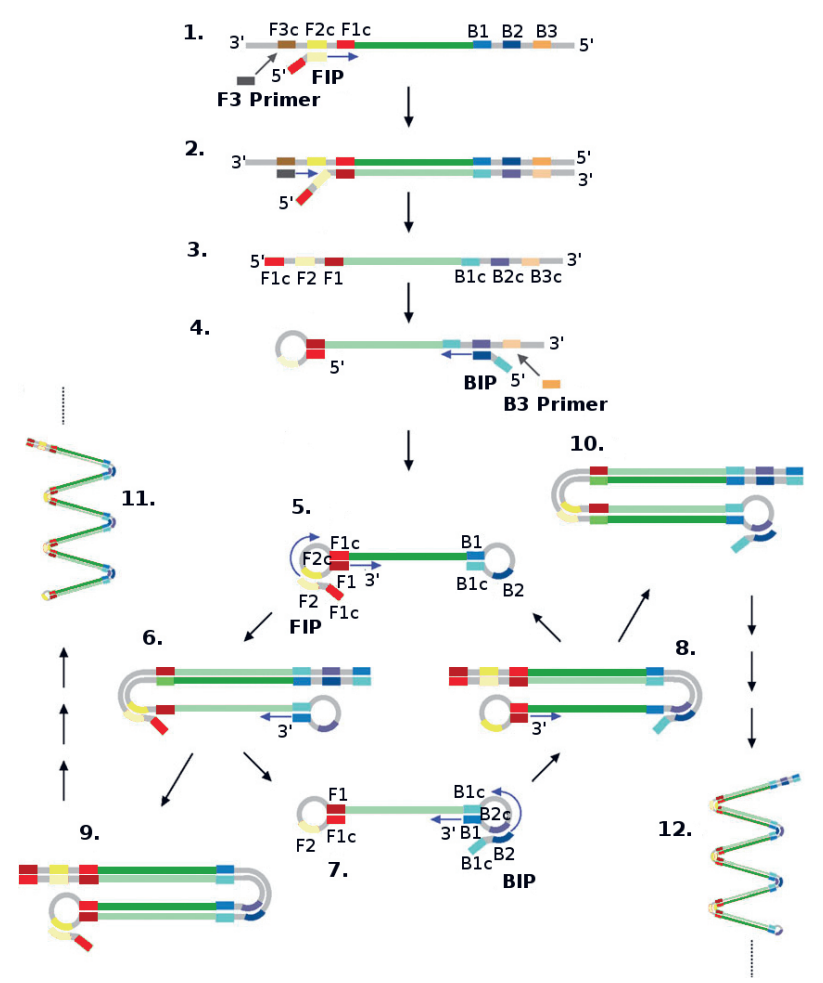

Abb. 1: Schema der LAMP Reaktion. Durch 4 verschiedene Primer, die an 6 unterschiedliche Regionen der Zielsequenz binden, und eine Strangverdrängungsreaktion wird die Hantelstruktur 5 gebildet. Anschließend wird die Sequenz durch selbstgeprimte Synthese und Strangverdrängung über einen Kreislauf vervielfältigt. Hierbei kommt es zur Bildung von unterschiedlich großen Produkten (Abbildung verändert nach [12]).

Als Nebenprodukt der Reaktion reichern sich Pyrophosphationen in großen Mengen an, die mit vorhandenen Magnesiumionen Komplexe bilden. Diese präzipitieren als weißer, deutlich sichtbarer Niederschlag und ermöglichen so eine Detektion von Reaktionsprodukten [13]. Tomita und Kollegen [12] konnten zeigen, dass durch den Einatz des Fluoreszenzfarbstoffes Calcein und dem Quencher Mangandichlorid eine Verbesserung der Produktdetektion möglich wird. Dabei werden Manganionen während der Amplifikation mit entstehenden Pyrophosphationen komplexiert und das anfängliche quenching des Calceins wird rückgängig gemacht. Die entstehende Fluoreszenz des Calceins wird dabei zusätzlich durch die Komplexierung von vorhandenen Magnesiumionen verstärkt (Abb. 2).

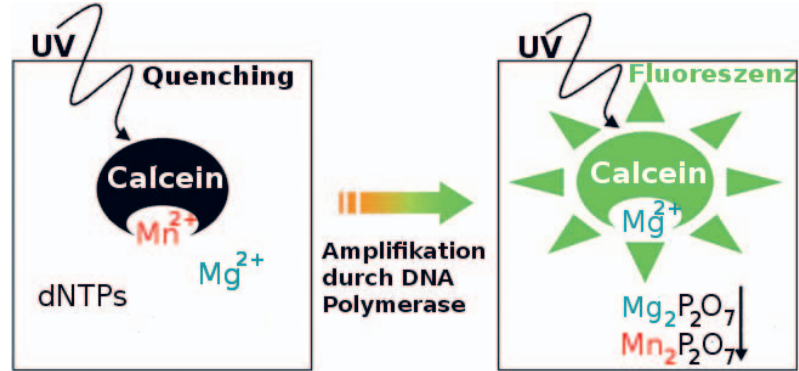

Abb. 2: Prinzip der Fluoreszenzreaktion zur direkten Produktdetektion mit Hilfe der LAMP-Methode. Zu Beginn der Reaktion ist das Calcein durch die Bindung von vorhandenem Mangan »gequenched . Durch die Reaktion der Polymerase bildet sich Pyrophosphat, das mit dem Mangan komplexiert. Hierdurch wird das »quenching« des Calceins rückgängig gemacht (Abbildung verändert nach [12]).

Die Sensitivität von LAMP ist vergleichbar mit der von PCR basierten Detektionsmethoden und konnte für verschiedene Pflanzenviren und -viroide bestimmt werden [14-18]. Im Folgenden wird die Detektion von PSTV mit Hilfe von LAMP und einer gekoppelten Fluoreszenzreaktion gezeigt.

\section{Material und Methoden}

PSTV infiziertes positive Material wurde vom DSMZ Braunschweig - Deutsche Sammlung für Mikroorganismen und Zellkultur (DSZMZ PV-No.: PV-0064) bezogen. Als Negativkontrolle dienten gesunde Kartoffelpflanzen, die in einem kleinen Gewächshaus bei Raumtemperatur und 10 Stunden Beleuchtung gehalten wurden. Primer für die LAMP Reaktion wurden mit Hilfe der Primer Explorer Software (http://primerexplorer.jpe) anhand der RefSeq Sequenz von PSTV (GenBank: NC_002030.1) konstruiert. Das Primerset besteht aus einem Forward Primer (F3), einem Backward Primer (B3), einem Forward Inner Primer (FIP) und einem Backward Inner Primer (BIP).

Für die RNA Extraktion wurde das RNeasy Plant Mini Kit (Quiagen) verwendet. Es wurden 100 mg Pflanzenmaterial in ein $1.5 \mathrm{ml}$ Reaktionsgefäß gegeben und gründlich mit einem Mikrohomogenisator in $450 \mu \mathrm{l}$ RLC Puffer zerkleinert. Das Lysat wurde anschließend in eine Qiashredder Spin Säule überführt und für 2 min bei $10.000 \mathrm{~g}$ zentrifugiert. Der Überstand wurde mit 0.5 Volumen Ethanol (99\%) vermischt und in eine RNeasy Spin Säule überführt. Einer Zentrifugation (1 min bei $8.000 \mathrm{~g}$ ) folgte ein Waschschritt mit 700 l RW1 Puffer bei $8000 \mathrm{~g}$ für $1 \mathrm{~min}$. Nach zwei Waschschritten mit 500 $\mu$ l RPE Puffer (gemischt mit 4 Volumenteilen Ethanol 
(99\%)) bei $8.000 \mathrm{~g}$ für 1 min und 2 min wurde die gereinigte RNA mit RNAse freiem Wasser durch Zentrifugation bei 8000 g für 1 min eluiert. Die Konzentration der RNA wurde mit einem Nanodrop Spectrophotometer bestimmt.

Die Reverse Transkription wurde in einem Volumen von $25 \mu$ durchgeführt. 500 ng RNA der aufgereinigten RNA Probe wurden mit $1 \mu \mathrm{M}$ des Backward Primers (B3) gemischt. Die Lösung wurde bei $90^{\circ} \mathrm{C}$ für 5 min denaturiert und für 5 min auf Eis gekühlt. 11 pl des Mastermixes wurden anschließend zur Reaktionslösung gegeben. Der Mastermix bestand aus 50 Units M-MLV Reverse Transkriptase RNase H Minus Point Mutant (Promega); 5 pl M-MLV RT 5x Reaktionspuffer (Promega); 0.5 mM dNTPs and 20 Units RNasin Ribonuklease Inhibitor (Promega). Die Reaktionslösung wurde bei $50^{\circ} \mathrm{C}$ für 60 min inkubiert. Inaktivierung der Reaktion erfolgte bei $70^{\circ} \mathrm{C}$ für $15 \mathrm{~min}$.

Die LAMP Reaktion wurde bei Reaktionstemperaturen von $64^{\circ} \mathrm{C}, 65^{\circ} \mathrm{C}, 66^{\circ} \mathrm{C}, 67^{\circ} \mathrm{C}$ und $68^{\circ} \mathrm{C}$ durchgeführt. Der Mastermix (Gesamtvolumen 24 ul) bestand aus 1.6 $\mathrm{\mu M}$ (jeder) der Inneren Primer FIP und BIP, 0.2 $\mu \mathrm{M}$ des Forward Primers (F3) und des Backward Primers (B3), $2.5 \mu \mathrm{l}$ 10x ThermoPol Reaktionspuffer (New England BioLabs), $1.2 \mathrm{mM}$ dNTPs, $2 \mathrm{mM}$ MgSO4, $0.8 \mathrm{M}$ Betaine, $1.5 \mathrm{mM} \mathrm{MnCl2,} 25 \mu \mathrm{M}$ Calcein (Merck) und 1 pl Ziel-DNA. Der Mastermix wurde bei $95^{\circ} \mathrm{C}$ für 5 min denaturiert. Nachdem die Temperatur für 5 min auf die Reaktionstemperatur reduziert wurde, wurden 8 Units Bst DNA Polymerase, large fragment (New England BioLabs) hinzugefügt. Die Amplifikation wurde für 1 Stunde durchgeführt. Die Reaktion wurde durch Inkubation bei $95^{\circ} \mathrm{C}$ für 2 min beendet. Nach der Inaktivierung wurden die Reaktionsgefäße bei Tages- und UV-Licht (Emissionswellenlänge $=312 \mathrm{~nm}$ ) betrachtet. Zusätzlich wurden die Reaktionsprodukte mit einer Gelelektrophorese bei $70 \mathrm{~V}$ für $40 \mathrm{~min}$ in einem $2.5 \%$ Agarosegel und 1x TAE Puffer aufgetrennt. Das Gel wurde in einer Ethidiumbromidlösung $(0.5 \mu \mathrm{g} / \mathrm{ml})$ gefärbt und unter UV-Licht betrachtet.

Die Real Time Detektion wurde mit einer Anregungswellenlänge von $483 \mathrm{~nm}$ und einer Emissionswellenlänge von $533 \mathrm{~nm}$ im LightCycler 480 (Roche) durchgeführt. Die Reaktionslösung wurde analog der LAMP Reaktion vorbereitet. Nach Zugabe der Bst DNA Polymerase wurde die Reaktionslösung in eine LightCycler Inkubationsplatte gegeben, mit Sealing Folie verschlossen und bei $65^{\circ} \mathrm{C}$ für 90 min inkubiert. Während der Reaktion wurde jede Minute die Fluoreszenz der Probe bestimmt. Die Reaktion wurde bei $95^{\circ} \mathrm{C}$ für 2 min gestoppt. Die Amplifikationsprodukte wurden mit einer Gelelektrophorese analysiert.

\section{Ergebnisse}

Es wurden RNA Proben von PSTV infiziertem Pflanzenmaterial und gesunden Kontrollproben verwendet. Die Reverse Transkription wurde mit dem PSTV-spezifischen B3 Primer durchgeführt.

Die Reaktion wurde zuerst ohne Denaturierung des Mastermixes und der cDNA durchgeführt. Hierbei konnte kein Reaktionsprodukt und kein Farbumschlag in der Reaktionslösung für die Positivprobe festgestellt werde (Daten nicht gezeigt). Nach einer Denaturierung von Mastermix und cDNA konnte ein weißer Niederschlag in der Positivprobe bei einer optimalen Reaktionstemperatur von $65^{\circ} \mathrm{C}$ festgestellt werden (Abb. 3a). Beobachtungen bei Tageslicht und UV-Licht zeigten einen deutlichen Farbumschlag (Abb. 3a) und ein starkes Fluoreszenzsignal (Abb. 3b) für die Positivprobe.
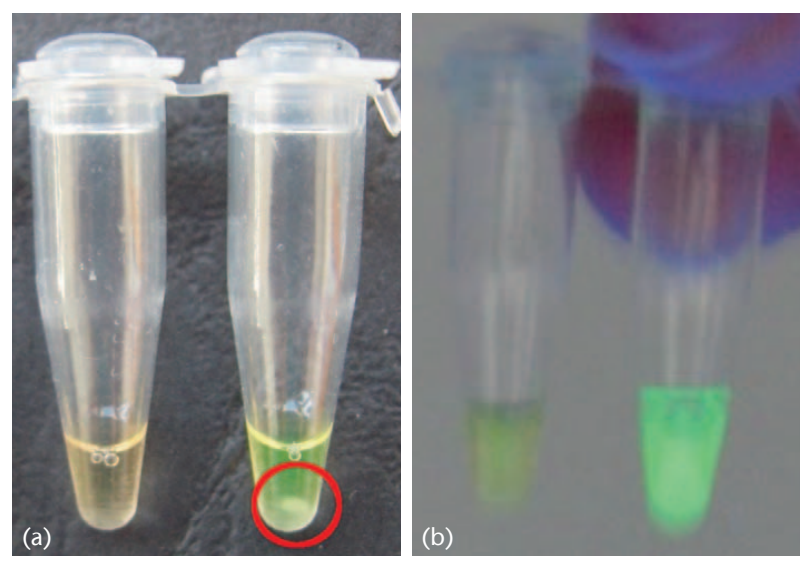

Abb. 3: Analyse der LAMP-Produkte bei (a) Tageslicht und (b) UV-Licht (links: nicht infizierte Probe, rechts: Positivmaterial). Im roten Kreis in Abbildung (a) ist der Mn/Mg-Pyrophosphat-Niederschlag zu erkennen.

Zusätzlich wurde eine Real Time Detektion mit Hilfe des LightCycler 480 durchgeführt. Hierbei wurde jede Minute die Fluoreszenz der Reaktionslösung ermittelt (Abb. 4). Es wurden Triplikate der Positivprobe und der Kontrollprobe verwendet. Zu Beginn der Reaktion zeigten alle Proben ein ähnliches Signal zwischen 180 und 220 Fluoreszenzeinheiten. Nach ca. 45 Minuten konnte für die Positivprobe ein linearer Anstieg von ca. 190 auf 1.200 Einheiten beobachtet werden. Die Kontrollprobe zeigte gleichzeitig einen nur sehr geringen Anstieg von ca. 50 Einheiten. Somit ergab sich nach 90 Minuten Re- 
aktionszeit ein Unterschied von ca. 800 Fluoreszenzeinheiten zwischen Positivmaterial und nicht infiziertem Material.

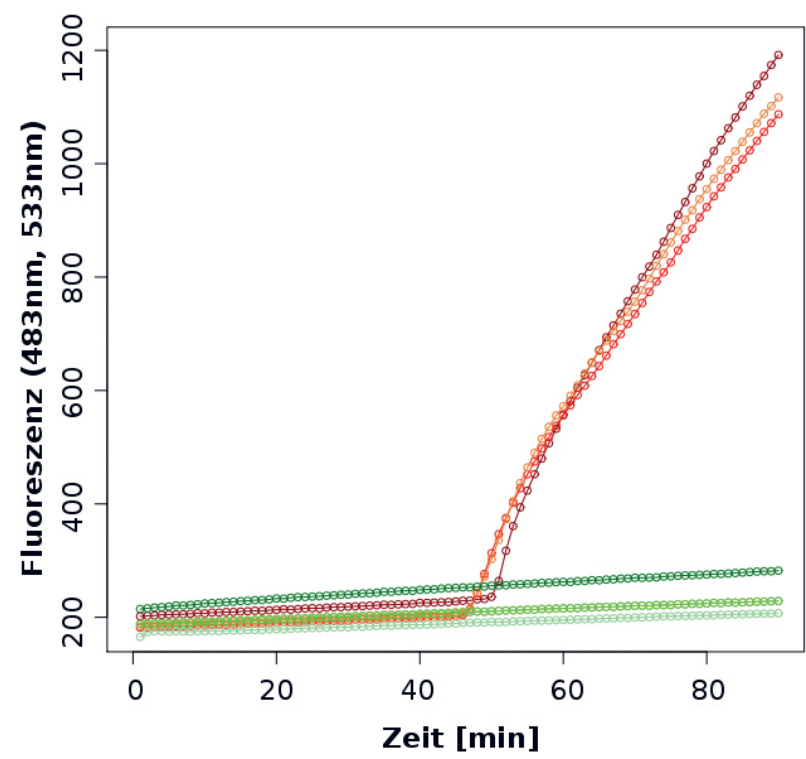

Abb. 4: Ergebnisse der Real Time Fluoreszendetektion (rot: Positivprobe, grün: nicht infizierte Probe)

Die Proben der Standardreaktion und der Real Time Detektion wurden zusätzlich mit einer Gelelektrophorese analysiert (Abb. 5). Für beide Methoden zeigten die Positivproben typische LAMP Produkte unterschiedlicher Größe. Zudem zeigten beide Positivproben identische Amplifikationsprodukte.

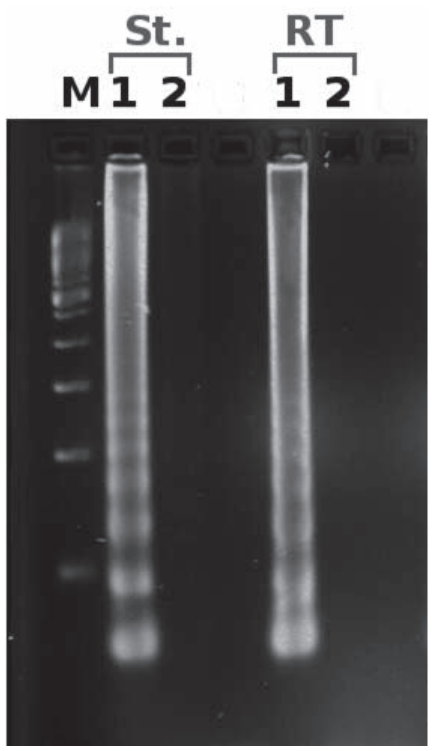

Abb. 5: Agarosegelelektrophorese der LAMP Produkte. Es sind jeweils Positivprobe (1) und nicht infizierte Probe (2) für die Standardreaktion (St.) und die Real Time Reakiton (RT) zu sehen (M: GeneRuler $\left.{ }^{\mathrm{TM}} 1 \mathrm{~kb}\right)$.

\section{Diskussion}

Das Potato spindle tuber viroid ist ein Pathogen das großen Schaden an Kartoffeln hervorrufen kann. Schnelle und sensitive Nachweismethoden werden immer wichtiger, um eine Verbreitung der Krankheit zu vermeiden. Obwohl RT-PCR eine sehr sensitive Detektion des Viroids ermöglicht, hat die Methode mehrere Nachteile. Sie ist sehr komplex in der Durchführung und zeitaufwendig. Zudem wird toxisches Ethidiumbromid für die Produktdetektion benötigt. Die Entwicklung von Real Time RT-PCR Methoden führen zwar zu einer Verringerung von Dauer und Aufwand des Nachweises, benötigen aber teure und anspruchsvolle Laborgeräte.

Die loop mediated isothermal amplification stellt hierbei eine sehr viel versprechende Alternative dar. Die isothermalen Reaktionsbedingungen ermöglichen eine sehr einfache Reaktion, für die nur ein günstiger Heizblock oder ein Wasserbad benötigt werden. Mehrere Ansätze zeigten, dass die Sensitivität dieser Methode mit der Sensitivität von PCR Reaktion vergleichbar ist $[14,16,17]$. Die Reaktion besitzt eine hohe Spezifität, da vier verschiedene Primer, die an 6 verschiedenen Regionen der Zielsequenz binden, verwendet werden. Während der Reaktion entstehende Pyrophosphationen, welche für eine gekoppelte Fluoreszenzreaktion genutzt werden können, ermöglichen eine sehr einfache Produktdetektion im Reaktionsgefäß ohne toxische Reagenzien.

Die typischen Reaktionsprodukte unterschiedlicher Größe konnten für die infizierte Probe nach einer Denaturierung beobachtet werden. Da PSTV durch starke Selbsthybridisierung (Faltung) charakterisiert ist, war diese notwendig, um die Bindung der Reaktionskomponenten zu ermöglichen. Es konnte eine optimale Reaktionstemperatur von $65^{\circ} \mathrm{C}$ bestimmte werden. Zusätzlich konnte durch einen sichtbaren Mn-Pyrophosphat Niederschlag der Quenchingeffekt für das zugegebene Calcein rückgängig gemacht werde. Die daraus resultierende starke Fluoreszenz ermöglichte somit die Identifikation von PSTV infizierten Proben mit dem bloßen Auge.

Die Real Time Detektion zeigte einen starken Signalanstieg nach $45 \mathrm{~min}$ für die infizierte Probe und unterstützt somit die vorhergehenden Ergebnisse. Der geringe Signalanstieg für die Kontrollprobe wurde wahrscheinlich durch die Bildung von kleinen Mengen an Primerdimeren hervorgerufen. Der große Unterschied von 800 Fluoreszenzeinheiten zwischen nicht infizierter und infizierter Probe ermöglicht hierbei trotzdem eine klare Unterscheidung zwischen positiver und negativer Probe. 


\section{Schlussfolgerung und Ausblick}

Wir schlagen die RT-LAMP als neue Nachweismethode für PSTV in Pflanzen vor. Im Vergleich zur RT-PCR handelt es sich hierbei um eine sehr schnelle und einfache Methode, für die nur einfache Laborgeräte benötigt werden. Somit kann die Methode in fast jedem Labor durchgeführt werden. Aufgrund dieser Vorteile schlagen wir weiterhin vor, dass die verantwortlichen Nationalen und Internationalen Autoritäten im Pflanzenschutz wie z. B. die European and Mediterranean Plant Protection Organization (EPPO) LAMP als alternative Nachweismethode für PSTV in ihren Richtlinien in Erwägung ziehen, sobald die Sensitivität der Methode bestätigt ist.

Forschungsergebnisse anderer Gruppen zeigen, dass die Bst Polymerase in der Lage ist LAMP unter Bedingungen durchzuführen bei denen die Taq Polymerase inhibiert wird. Beispiele sind Reaktionen mit extrahierter DNA aus Fäkalien [19] und mit Blut geblottetem Filterpapier [20].

Für die Zukunft scheint auch eine Anwendung des LAMP Nachweises im Pflanzensaft, trotz vorhandener inhibierender Substanzen wie z. B. Polysaccharide oder Phenolkomponenten, möglich. Mit weiteren Verbesserungen der Methode ist möglicherweise die Anwendung im Feld denkbar.

Die wesentlichen Experimente wurden im Rahmen der Masterarbeit der Erstautorin im Labor für Molekularbiologe und Funktionelle Genomik der Technischen Hochschule Wildau [FH] durchgeführt. Wir danken Prof. Dr. rer. oec. habil. Lothar Brunsch für die Betreuung des Teils dieser Arbeit, die sich mit wirtschaftlichen Aspekten auseinander gesetzt hat.

Wir danken der Firma Elsner pac ${ }^{\circledR}$ Jungpflanzen GbR (Dresden) für ihre Unterstützung und dem Landesamt für Verbraucherschutz, Landwirtschaft und Flurneuordnung (LVLF), Brandenburg für die Hilfe bei der Schaffung von regulatorischen und administrativen Voraussetzungen.

\section{Quellen}

[1] Sänger H L, Gross H J, Domdey H, Lossow C, Jank P, Raba M, Alberty H: Nucleotide sequence and secondary structure of potato spindle tuber viroid. Nature 1987, 273:203-208.

[2] Singh R P, Kurz J: RT-PCR analysis of PSTVd aphid transmission in association with PLRV. Canadian Phytopathological Society 1997, 19: 418-424.

[3] Seigner L, Kappen M, Huber C, Kistler M, Köhler D: First trials for transmission of Potato spindle tuber viroid from ornamental Solanaceae to tomato using RT-PCR and an mRNA based internal positive control for detection. J Plant Dis Prot 2008, 15:97-101.

[4] Podleckis E V, Hammond R W, Hurtt S S, Hadidi A: Chemiluminescent detection of potato and pome fruit viroids by digoxigeninlabeled dot blot and tissue blot hybridization. J Virol Methods 1993, 43:147-155.

[5] European and Mediterranean Plant Protection Organization (EPPO): EPPO Standards: Diagnostic protocols for regulated pests PM 7/33. EPPO Bulletin 2004, 34:257-269.

[6] Weidemann H L, Buchta U: A simple and rapid method for the detection of potato spindle tuber viroid (PSTVd) by RT-PCR. Potato Res 1998, 41:1-8.

[7] Nie X, Singh R P: Detection of multiple potato viruses using an oligo(dT) as a common cDNA primer in multiplex RT-PCR. J Virol Methods 2000, 86:179-185.

[8] Ragozzino E, Faggioli F, Barba M: Development of a one tube-one step RTPCR protocol for the detection of seven viroids in four genera: Apscaviroid, Hostuviroid, Pelamoviroid and Pospiviroid. J Virol Methods 2004, 121:25-29.

[9] Shamloul A M, Faggioli F, Keith J M, Hadidi A: A novel multiplex RT-PCR probe capture hybridization (RT-PCR-ELISA) for simultaneous detection of six viroids in four genera: Apscaviroid, Hostuviroid, Pelamoviroid, and Pospiviroid. J Virol Methods 2002, 105:115-121.

[10] Boonham N, González Pérez L, Mendez M S, Lilia Peralta E, Blockley A, Walsh K, Barker I, Mumford R A: Development of a real-time RT-PCR assay for the detection of Potato spindle tuber viroid. J Virol Methods 2004, 116:139-146.

[11] Notomi T, Okayama A, Masubuchi H, Yonekawa T, Watanabe K, Amino N, Hase T: Loop-mediated isothermal amplification of DNA. Nucl Acids Res 2000, 12:E63-e63.

[12] Tomita N, Mori Y, Kanda H, Notomi T: Loop-mediated isothermal amplification (LAMP) of gene sequences and simple visual detection of products. Nat Protoc 2008, 3:877-882.

[13] Mori Y, Nagamine K, Tomita N, Notomi T: Detection of LoopMediated Isothermal Amplification Reaction by Turbidity Derived from Magnesium Pyrophosphate Formation. Biochem Bioph Res Co 2001, 289:150-154.

[14] Boubourakas I N, Fukuta S, Kyriakopoulou P E: Sensitive and rapid detection of peach latent mosaic viroid by the reverse transcription loop-mediated isothermal amplification. J Virol Methods 2009, 160:63-68.

[15] Fukuta S, Kato S, Yoshida K, Mizukami Y, Ishida A, Ueda J, Kanbe $\mathrm{M}$, Ishomoto Y: Detection of tomato yellow leaf curl virus by loop-mediated isothermal amplification reaction. J Virol Methods 2003, 112:35-40.

[16] Fukuta S, Niimi Y, Oishi Y, Yoshimura Y, Anai N, Hotta M, Fukaya M, Kato T, Oya T, Kambe M: Development of reverse transcription loop-mediated isothermal amplification (RT-LAMP) method for detection of two viruses and chrysanthemum stunt viroid. Annual Report of the Kansai Plant Protection Society 2005, 47:31-36. 
[17] Varga A, James D: Use of reverse transcription loop-mediated isothermal amplification for the detection of plum pox virus. J Virol Methods 2006, 138:184-190.

[18] Nie X: Reverse Transcription Loop-Mediated Isothermal Amplification of DNA for Detection of Potato virus Y. Plant Dis 2005, 89:605-610.

[19] Bakheit M A, Torra D, Palomino L A, Thekisoe O M, Mbati P A, Ongerth J, Karanis P: Sensitive and specific detection of Cryptosporidium species in PCR-negative samples by loop-mediated isothermal DNA amplification and confirmation of generated LAMP products by sequencing. Vet Parasitol 2008, 158:11-22.

[20] Alhassan A, Thekisoe O M, Yokoyama N, Inoue N, Motloang M Y, Mbati P A, Yin H, Katayama Y, Anzai T, Sugimoto C, Igarashi I: Development of loop mediated isothermal amplification (LAMP) method for diagnosis of equine piroplasmosis. Vet Parasitol 2007, 143:155-160.

\section{Autoren}

Katja Schulze M.Sc

Biosystemtechnik/Bioinformatik

Molekularbiologie und Funktionelle Genomik

Technische Hochschule Wildau [FH]

katja.schulze@th-wildau.de

Dr. Jörn Glökler

Abteilung für Wirbeltiergenomik

Max Planck Institut für Molekulare Genetik

Berlin

Dr. Marianne Kadolsky

Elsner pac ${ }^{\circledast}$ Jungpflanzen GbR

Dresden

\section{Prof. Dr. Marcus Frohme}

Molekularbiologie und Funktionelle Genomik Technische Hochschule Wildau [FH]

marcus.frohme@th-wildau.de 